\title{
The system design for calibrating acoustic Doppler current meter in the laboratory
}

\author{
Hongxu Jiang ${ }^{1,2,3}$, Yalin $\mathrm{Li}^{1,2,3}$, Yongwei Liu ${ }^{1,2,3^{*}}$, and Dejiang Shang ${ }^{1,2,3}$ \\ ${ }^{1}$ Acoustic Science and Technology Laboratory, Harbin Engineering University, Harbin, China, 150001 \\ ${ }^{2}$ College of Underwater Acoustic Engineering, Harbin Engineering University, Harbin, China, 150001 \\ ${ }^{3}$ Key Laboratory of Marine Information Acquisition and Security (Harbin Engineering University), Ministry of Industry and \\ Information, Harbin, China, 150001
}

\begin{abstract}
The Acoustic Doppler Current meter is a most commonly device for the velocity measurement of the seawater in the ocean. We had designed an equipment to calibrate the Acoustic Doppler Current meter in the laboratory, which is mainly composed of a vertical circulating water tank and a system of Laser Doppler Velocimetry. The vertical circulating water tank is the key device of the calibration equipment. The optimization of flow field is carried out by the software, CFD. The optimization of structure intensity, modal analysis and low noise is carried out by the software, ANSYS. We had also used the propeller map to design the power section of the water tank. Through the comparison of the velocity of the flow between Acoustic Doppler Current meter and LDV at the same position in the working section of the water tank, the former can be calibrated, because the wavelength of optics is much less than that of acoustics in the water. After the experimental test, we found that when the fluid flows in the vertical circulating water tank, there exists no resonance phenomenon and the velocity of the flow in the working section is approximately proportional to the rotation speed of the propeller.
\end{abstract}

\section{Introduction}

In 1963, Koczy proposed the use of acoustic Doppler principle for velocity profile measurement[1]. After more than 50 years, the acoustic Doppler current meter has been continuously developed and become the main equipment in current international flow velocity measurement[2]. The calibration of an acoustic Doppler current meter is the necessary step in the flow velocity measurement. The current calibration is mainly carried out in the external waters, which is greatly affected by environmental factors. Therefore, the calibration system of the Doppler current meter in the laboratory is of great significance to improve the measurement accuracy.

The calibration system of Doppler current meter in the laboratory designed in the paper is mainly composed of a vertical circulating water tank and a laser Doppler speed measurement device. The circulating water tank is a hydrodynamic experimental device that allows water to flow and the model is placed in flowing water. In the circulating water tank, the water circulates at a certain speed by means of the power system, and the working section can be tested for a long time, which is convenient for velocity measurement[3]. The stability of the flow field of the working section of the circulating water tank will greatly affect the calibration accuracy. The hydrodynamic noise caused by the flow of water in the circulating water tank will cause serious background noise interference to the measurement instrument, so that the optimization of flow field stability and low noise is particularly important [4]. In addition, in the process of the water flowing through the circulating water tank, the flow velocity and pressure of the water tank change due to the change of the radius of the water tank, the corner, etc., must be considered, which cause the vibration of the tank body, radiates noise, and has a certain impact on the life and safety of the circulating water tank[5]. Therefore, the design of the circulating water tank should avoid resonance. According to the principle of the laser Doppler, the LDV uses the scattering particles in the measurement body to scatter the incident light and detect the frequency shift of the scattered light through the photo-detector. According to this frequency shift, the velocity of the moving object can be calculated, which has the advantages of fast dynamic response, high spatial resolution, large measurement range, non-contact measurement and good real-time performance[6]. As shown in Figure 1, the flow velocity of the working section of the circulating water tank is measured by LDV, and compared with the measured flow velocity by the acoustic Doppler current meter to complete the calibration of the acoustic Doppler current meter. The basic principle of the calibration system is that the frequency of the optical wave in the water is higher than that of the sound wave, the wavelength is short, and the measurement accuracy is higher. The measurement result of the optical device is used as the criterion to verify the measurement accuracy of the acoustic device.

* Corresponding author: liuyongwei3000@hrbeu.edu.cn 


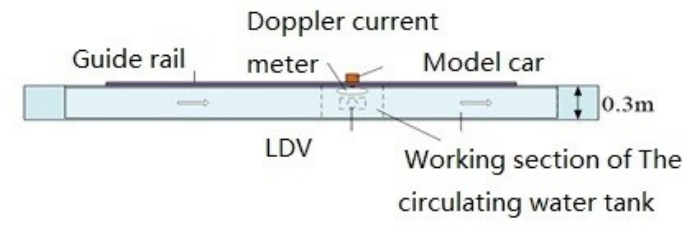

Fig. 1. Doppler current meter calibration device.

This paper introduces the structural design of each part of the vertical circulating water tank. Through the CFD and finite element calculation, the flow field optimization and low noise design of the circulating water tank are completed. The flow velocity of the working section of the circulating water tank at different speeds is measured by laser Doppler velocimetry, and the relationship between the flow velocity of the working section of the circulating water tank and the speed of the propeller is obtained, so as to quickly obtain the required working water flow velocity when the Doppler current meter is calibrated. That how the acoustic Doppler current meter calibration system works is validated. The research results in this paper fill with the blank of calibrating the Doppler current meter device in the laboratory, and also provide reference for the structure and low noise design of the circulating water tank.

\section{Design of the vertical circulating water tank}

\subsection{Structure design of the circulating tank}

The flow velocity of the working section of the vertical circulating water tank is $0.01 \mathrm{~m} / \mathrm{s}-0.3 \mathrm{~m} / \mathrm{s}$, which can carry out the calibration of the Doppler current meter at low flow velocity. The vertical circulating water tank includes the working section, rectifying and constricting section, corner section, power section and diffusion section. The overall dimension of the circulating water tank is $2.46 \times 0.2 \times 1.17 \mathrm{~m}$. Fig. 2 is a schematic diagram of the plane structure of the vertical circulating water tank.

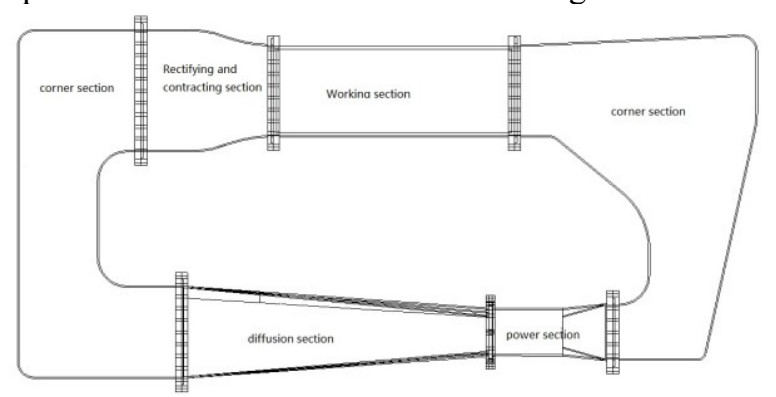

Fig. 2. The schematic structure of the circulating water tank.

\subsection{The optimization design of flow field in the circulation water tank}

As a whole, the flow field of the circulating water tank is relatively uniform. The contraction section of the rectifying section has a good effect on the upstream flow, and the flow field in the working section is uniform and stable. However, the four corner segments, especially the first corner segment downstream of the working section, have a relatively serious phenomenon of flow separation. Four corner sections need to be optimized for the flow field, which is required to improve the flow stability and reduce hydrodynamic noise. According to the design experience of wind tunnel, the method of the installation of the deflectors is proposed.

There are three common types of deflectors; one is the quarter circle bent plate, which is the earliest used. Later, Klein [7] and others added a straight section of appropriate length to the trailing edge to form an arcshaped linear deflector. B. Eck [8] added thickness to the quarter arc deflector to form the most common double arc airfoil deflector. The corner section is established according to the second corner section of the circulating water tank, $0.3 \mathrm{~m}$ high and $0.3 \mathrm{~m}$ wide. The arrangement types of deflectors are evenly distributed, the inner and outer sparse rows arranged in the arithmetic progression; the inner sparse and dense outer rows arranged in the arithmetic progression. A total of 9 sets of models were used for the three types of layout, and compared with the model without the deflectors to validate the effect of the suppression of flow separation.

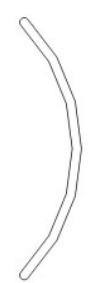

(a)Circle

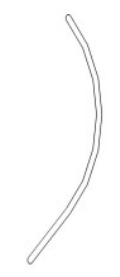

(b) Straight line

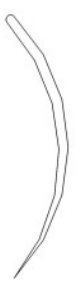

(c) Double circle airfoil
Fig. 3. Three types of deflectors.

The structure of 10 groups of models is divided by the ICEM, and imported into the software, Fluent, for the calculation. The inlet boundary condition is set as the velocity inlet; the velocity is $0.3 \mathrm{~m} / \mathrm{s}$. The outlet is the pressure outlet. The rigid wall condition, the turbulence model, and the coupling between the pressure and velocity are selected in the SIMPLE method. The control equation diffusivity is the format of central difference, and the convection term is the QUICK discrete format.

Fig. 4 is the flow field streamline diagram of various types of deflectors and arrangements, and Table 1 is the calculated standard deviation of the outlet velocity. It is observed that there is a serious flow separation in the corner section when the deflector is not provided. After the deflector is set up, the phenomenon of flow separation is controlled. According to the calculation results in Table 1, it can be seen that the arrangement of the double-circle airfoil in the inner and outer sparse format has the optimum uniformity of the outlet velocity.

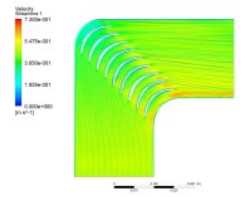

(a) Arc Uniform

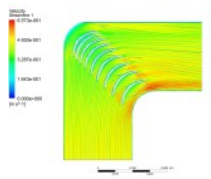

(c) Arc outer dense

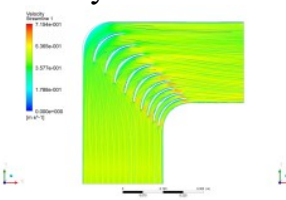

(b) Arc inner dense

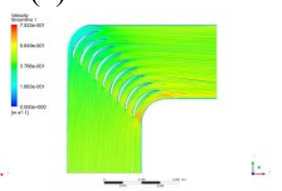

(d) Arc airfoil uniform 


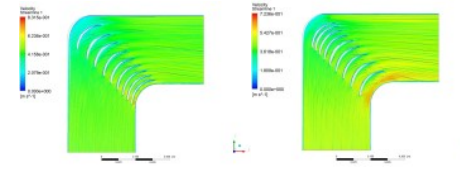

(e) Arc-airfoil inner dense (f) Arc airfoil outer dense
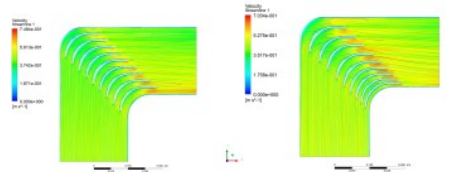

(g) Arc straight edge uniform (h) Arc straight edge inner dense

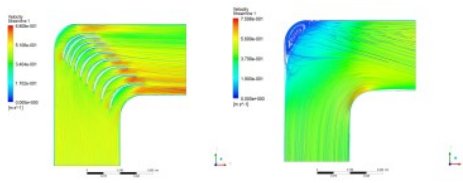

(i) Arc straight edge outer dense (j) No baffle

Fig. 4. The streamline diagram at corner.

Table 1. Standard deviation of outlet velocity of different deflector models.

\begin{tabular}{|c|c|}
\hline Arrangement & Speed standard deviation \\
\hline No deflector & 0.14547 \\
\hline Arc Uniform & 0.10418 \\
\hline Arc inner dense & 0.09810 \\
\hline Arc outer dense & 0.09942 \\
\hline Arc airfoil uniform & 0.10184 \\
\hline Arc airfoil inner dense & 0.09831 \\
\hline Arc airfoil outer dense & 0.10113 \\
\hline Arc straight edge uniform & 0.1183 \\
\hline Arc straight edge inner dense & 0.10869 \\
\hline Arc straight edge outer dense & 0.12462 \\
\hline
\end{tabular}

Fig. 5 is the vector diagram of the arrangement and the non-conduit outlet velocity, and it can be seen that when there is no deflector, there is a serious velocity deflection at the exit and secondary flow perpendicular to the main flow. This phenomenon is eliminated after the installation of the double-arc airfoil inner and outer sparse deflector, which will greatly enhance the uniformity of the flow field in the working section and reduce the hydrodynamic noise.

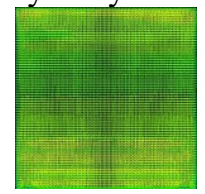

(a) Arc airfoil inner dense

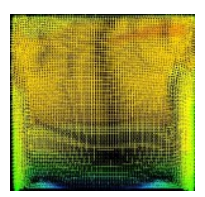

(b) No baffle
Fig. 5. The vector of outlet velocity.

\subsection{The calculation of circulating water tank}

The circulating water tank uses a propeller to drive the water flow. Therefore, the main reason for the steadystate forced vibration of the tank body is the periodic excitation force caused by the rotation of the propeller. The excitation force caused by the propeller can be divided into two categories: One type is the axial frequency excitation force, that is, the excitation frequency of the propeller is equal to the first-order excitation force of the propeller's shaft speed, which is caused by the mechanical imbalance of the propeller. The other type is the high-order excitation force, of which the excitation frequency is equal to the paddle speed, $n$, multiplied by the blade number, $Z$, or the blade multiple, called the excitation force of blade frequency or the excitation force of lobulation frequency, which is caused by the propeller in an uneven flow field. Therefore, the excitation force of the circulating water tank needs to consider the axial frequency, the blade frequency and the multiple frequencies. The frequency of propeller shaft is calculated by the equation (1).

$$
f=\frac{N}{60}
$$

where $\mathrm{N}$ is the paddle speed. The flow velocity of the working section of the circulating water tank is $0.3 \mathrm{~m} / \mathrm{s}$, and the speed of the propeller shaft is $466.67 \mathrm{r} / \mathrm{min}$. The frequency of the propeller shaft on the condition is $f=7.78 \mathrm{~Hz}$. Since the propeller is a three-bladed paddle, the frequency of propeller blade is $23.33 \mathrm{~Hz}$ and the frequency of the lobes is $46.67 \mathrm{~Hz}$.

The flange connections of the circulating water tank are removed, a simplified calculation model of the circulating water tank is established by the software, ANSYS Workbench, to calculate the characteristic frequency of the tank. If the case that no constraint is added and the bottom of the tank is added, the modal calculation is performed on the circulating water tank. The six-order vibration frequency of the circulating water tank is obtained as shown in Table 2 .

Table 2. The characteristic frequency of the circulating water tank.

\begin{tabular}{|c|c|c|}
\hline Order & $\begin{array}{c}\text { The natural frequency } \\
\text { of the tank with the } \\
\text { bottom added } / \mathbf{H z}\end{array}$ & $\begin{array}{c}\text { Unconstrained } \\
\text { characteristic } \\
\text { frequency } / \mathbf{H z}\end{array}$ \\
\hline 1 & 122.7 & 0 \\
\hline 2 & 125.06 & 0.0039 \\
\hline 3 & 174.02 & 0.0045 \\
\hline 4 & 194.79 & 0.0068 \\
\hline 5 & 254.37 & 0.0794 \\
\hline 6 & 263.19 & 0.0817 \\
\hline
\end{tabular}

When the frequency of propeller excitation force is equal or close to the characteristic frequency of the circulating water tank, the tank coupling resonance occurs, and the radiation noise is generated. The $\pm 10 \%$ interval of the characteristic frequency of the circulating water tank is taken as the resonance interval; the resonance frequency range is calculated. Compared with the frequency of the propeller excitation force, it is found that in the presence of water or no water, the characteristic frequency of the circulating water tank with the bottom constraint is much larger than the 
frequency of propeller excitation. The characteristic frequency of the circulating water tank without the constraint is much smaller than frequency of the propeller excitation. Therefore, the circulating water tank does not undergo coupling resonance during the operation.

\section{Flow velocity measurements}

If the variation law of the flow velocity of the working section of the circulating water tank and the rotation speed of the propeller is obtained, and the working principle of the acoustic Doppler current meter calibration system is validated, the required water flow velocity of the working section can be quickly obtained, when the Doppler current meter is calibrated. The flow velocity of the working section of the circulating water tank under different propeller's speeds is measured by the LDV. The relationship between the rotation speed of the propeller and the flow velocity of the working section was obtained.

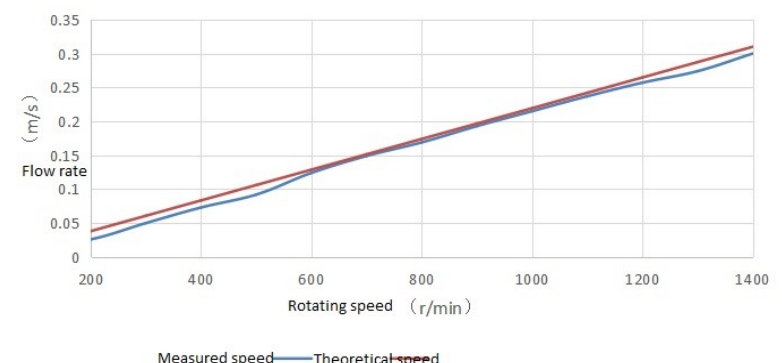

Fig. 6. The relationship between the speed of the propeller and the flow velocity of the working section.

Theoretically, the flow velocity of the working section of the circulating water tank is linear with the rotational speed of the propeller. The measured curve in Figure 6 is verified by the theoretical calculation results. Due to the local loss of the circulating water tank, the measured flow velocity in the working section is smaller than the theoretical one. When the acoustic Doppler current meter is calibrated, the flow velocity can be quickly adjusted through the relationship.

\section{Conclusions}

A Doppler current meter calibration system in the laboratory is designed, which consists of a small vertical circulating water tank and a laser Doppler velocity measurement device (LDV). The flow velocity of the working section of the small vertical circulating water tank is $0.01 \mathrm{~m} / \mathrm{s}-0.3 \mathrm{~m} / \mathrm{s}$, which can realize the calibration of the Doppler current meter at low flow velocity. The overall dimension is $2.46 \times 0.2 \times 1.17 \mathrm{~m}$, and the dimension of the working section is $0.8 \times 0.2 \times 0.3 \mathrm{~m}$. Through the numerical simulation, the inner and outer sparse deflectors arranged in the arithmetic progression are placed in the corner section. By the comparison between the frequency of the circulating water tank with or without constraints, the characteristic frequency and the frequency of the excitation force of the propeller, it is found that the circulating water tank does not resonate.
By the LDV to measure the flow velocity, it is found that the flow velocity of the working section of the circulating water tank is approximately linear with the rotation speed of the propeller, which is in accordance with the law of theoretical calculation. However, due to the local loss of the circulating water tank, the measured flow velocity of the working section is less than the theoretical value. The results of the paper can provide some reference for the design of vertical circulating water tanks with low noise and high stability of the flow field.

The work has been supported by the National Key Research Development Project, No.2016YFF0200906, Heilongjiang Province Foundation, No.GX17A015, and China Postdoctoral Science Foundation Funded Project, No.2017M611358. Elsewhere, the work has been supported by the major project from Acoustic Science and Technology Laboratory, No.9140C200104140C20003, and also been funded the steady support plan from Acoustic Science and Technology Laboratory (Grant No. SSJSWDZC2018005). Furthermore, the work has been funded by the project from Key Laboratory of Acoustic Stealth (Grant No. 614220405011706), the Fundamental Research Funds for Central universities (Grant No. HEUCF180503), and also funded by the China Scholarship Council, No.201706685061.

\section{References}

1. Huhta C, Ward C, Current Measurement Technology, 35-39(2003)

2. Lowell N S, Walsh D R, Pohlman J W, Current Waves and Turbulence Measurement. 1-7(2015)

3. Emschermann, Archiv Für Hydrobiologie, (108):425438(1987)

4. FU M, GENG H, XING Y, Heavy Machinery, 9092(2010)

5. Gladysz S, Rui B, Hou W, Society of Photo-Optical Instrumentation Engineers. 6(2017)

6. Wayner E A, Kovach N L, Journal of Cell Biology. 116(2):489-497(1992)

7. Lee S H, Park C M, Yong M S, et al. Physics, 2009.

8. Budil K S, Lasinski B, Edwards M J, et al. Physics of Plasmas. 8(5):2344-2348(2001) 